05

\title{
Резистивные состояния композитных сверхпроводников при крипе магнитного потока
}

\author{
(C) В.P. Романовский \\ Национальный исследовательский центр „Курчатовский институт“, \\ 123182 Москва, Россия \\ e-mail: romanovskii@aol.com
}

(Поступило в Редакцию 21 июня 2016 г.)

\begin{abstract}
Изучено влияние крипа магнитного потока на формирование резистивных состояний композитных сверхпроводников с учетом их саморазогрева. Полученные результаты сопоставлены с расчетами, выполненными в рамках существующей теории тепловой стабилизации, основанной на модели скачкообразного перехода из сверхпроводящего состояния в нормальное. Показано, что в широком диапазоне изменения температуры сверхпроводника эта модель приводит к завышенным значениям эффективного электрического сопротивления композита. Как следствие его стабильного саморазогрева, используемые в существующей теории тепловой стабилизации понятия о критическом токе, определяющем максимально допустимое значение транспортного тока, и о температуре резистивного перехода, после превышения которой транспортный ток начинает делиться между сверхпроводником и матрицей, при крипе магнитного потока теряют физический смысл. В результате границы теории тепловой стабилизации композитных сверхпроводников могут быть расширены, если ее использовать для описания устойчивых состояний при токах, которые выше условно заданного значения критического тока композита.
\end{abstract}

DOI: 10.21883/JTF.2017.04.44313.1943

Исследование макроскопических механизмов формирования устойчивых состояний низко- и высокотемпературных сверхпроводников, определение условий возникновения и распространения неустойчивостей различной природы представляют собой актуальные проблемы физики сверхпроводящих сред. Значительную роль в формулировке принципов, обеспечивающих сохранение работоспособности токонесущих элементов сверхпроводящих магнитных систем при действии внешних тепловых возмущений, играет теория тепловой стабилизации композитных сверхпроводников, состоящих из сверхпроводящих жил, помещенных в несверхпроводящую матрицу. Ее основные положения были сформулированы для низкотемпературных сверхпроводящих композитов в рамках модели скачкообразного перехода из сверхпроводящего состояния в нормальное (так называемая модель критического состояния МКС), которая позволила не только упростить используемые методы анализа, но и выписать аналитические критерии тепловой стабильности сверхпроводящего состояния [1-3]. Эта модель также широко используется и при описании тепловых процессов в композитах на основе высокотемпературных сверхпроводников (см., например, [4-6]). В рамках данного приближения предполагается, что транспортный ток равномерно распределен по сечению композита, а его значения в сверхпроводнике и матрице определяются мгновенными значениями температуры сверхпроводника. Возникающие при этом задачи сводятся к решению уравнения теплопроводности с нелинейной зависимостью источника тепловыделения от температуры. А именно согласно МКС, диссипация энергии в сверхпроводящем композите начинается только тогда, когда его температура превысит так называемую температуру резистивного перехода $T_{c s}$, при которой транспортный ток равен критическому току сверхпроводника. Соответственно при $T>T_{c s}$ ток начинает перераспределяться между сверхпроводником и матрицей, и после нагревания композита выше критической температуры сверхпроводника $T_{c B}$ весь ток течет только по матрице. Если плотность критического тока в сверхпроводнике линейно убывает с температурой, то температурная зависимость мощности джоулева тепловыделения в сверхпроводящем композите, согласно МКС, записывается как [1-3]

$$
\begin{aligned}
& G_{\mathrm{CSM}}(T)=\frac{J^{2} \rho_{m}(T, B)}{1-\eta} \\
& \times\left\{\begin{array}{l}
1, \quad T>T_{c B}, \\
\left(T-T_{c s}\right) /\left(T_{c B}-T_{c s}\right), \quad T_{c s} \leq T \leq T_{c B}, \\
0, \quad T<T_{c s}=T_{c B}-\left(T_{c B}-T_{0}\right) J /\left(\eta J_{c 0}\right) .
\end{array}\right.
\end{aligned}
$$

Здесь $J-$ плотность транспортного тока, $\eta-$ коэффициент заполнения композита сверхпроводником, $\rho_{m}-$ удельное электрическое сопротивление матрицы, $T_{0}$ температура хладагента, $J_{c 0}$ и $T_{c B}-$ критическая плотность тока сверхпроводника при температуре хладагента и его критическая температура в заданном внешнем магнитном поле соответственно.

Однако МКС описывает тепловые состояния композита в предположении, что перераспределение тока между сверхпроводником и матрицей происходит мгновенно, так как предполагается, что сопротивление сверхпроводника при достижении критической плотности тока меняется скачком от нулевого значения до бесконечно 
большого. В то же время композитные сверхпроводники как на основе низкотемпературных, так и высокотемпературных материалов в силу многих причин имеют непрерывно нарастающую вольт-амперную характеристику (BAX). В частности, конечное напряжение, возникающее в сверхпроводнике, может быть обусловлено объемной неоднородностью свойств сверхпроводника, термоактивированным движением вихрей. В последнем случае, известном как крип магнитного потока, величина энергии активации вихрей определяет вид ВАХ сверхпроводника. При этом зависимость потенциального барьера пиннинга от тока как у низкотемпературных, так и у высокотемпературных сверхпроводников может иметь нелинейность логарифмического типа $[7,8]$. В этом случае их $\mathrm{BAX}$ описываются степенным уравнением вида $E(J)=E_{c}\left(J / J_{c}\right)^{n}[2]$. Здесь $J_{c}-$ температурно-полевая зависимость критической плотности тока сверхпроводника, определенная при априори заданном значении напряженности электрического поля $E_{c}, n-$ параметр крипа, зависящий от свойств сверхпроводника. Другими словами, композитные сверхпроводники находятся в резистивном состоянии при любой конечной плотности введенного тока. В результате дифференциальное сопротивление сверхпроводника не только изменяется непрерывно, но оно может быть меньше удельного электрического сопротивления матрицы в соответствии с особенностями нарастания BAX сверхпроводника. Между тем полный анализ влияния характера нелинейности ВАХ на диссипативные явления в сверхпроводящих композитах, а значит и на условия их тепловой стабилизации, не выполнялся. Как следствие, ряд фундаментальных положений существующей теории тепловой стабилизации комбинированных сверхпроводников (и в первую очередь понятие о предельно допустимом токе, о механизме деления тока) сформулированы без учета нелинейных эффектов саморазогрева сверхпроводника с реальной BAX. В связи с этим в настоящей работе обсуждаются физические особенности джоулевой диссипации энергии, которые неизбежны в сверхпроводящих композитах с непрерывно нарастающей BAX и лежат в основе реальных процессов возникновения и развития в них тепловых неустойчивостей. Несмотря на то, что ниже рассмотрен высокотемпературный сверхпроводник, для которого характерен гигантский крип магнитного потока, сформулированные общие выводы справедливы и для низкотемпературных сверхпроводников.

Рассмотрим сверхпроводящий композит с площадью поперечного сечения $S$. Будем полагать, что он находится в постоянном магнитном поле $B$ и по нему течет постоянный ток с плотностью $J$. Предположим также, что:

- индуцированные током температура $T$ и напряженность электрического поля $E$ постоянны по сечению композита;

- между сверхпроводником и матрицей имеется идеальный тепловой и электрический контакты;
- объемная доля сверхпроводника, равномерно распределенного по композиту, равна $\eta$;

- $E(J)$ характеристика сверхпроводника описывается степенным уравнением, в котором изменение критической плотности тока с температурой может быть аппроксимировано линейной зависимостью вида

$$
J_{c}=J_{c 0}\left(T_{c B}-T\right) /\left(T_{c B}-T_{0}\right) .
$$

В рамках данных предположений температурная зависимость джоулева тепловыделения $G(T)=E J$, неизбежного для композитных сверхпроводников с реальной BAX при любом конечном значении введенного тока, является решением следующей системы уравнений:

$$
\begin{gathered}
E=E_{c}\left[J_{s c} / J(T, B)\right]^{n}=J_{m} \rho_{m}(T, B), \\
J=\eta J_{s c}+(1-\eta) J_{m} .
\end{gathered}
$$

Здесь $J_{s c}$ и $J_{m}-$ плотности токов, протекающих в сверхпроводящей части композита и в ее матрице соответственно. Тогда, учитывая равенство (2), найдем

$$
\begin{aligned}
& G(T)= \\
& \left\{\begin{array}{l}
\eta J_{c 0} E\left(1-\frac{T-T_{0}}{T_{c B}-T_{0}}\right)\left(\frac{E}{E_{c}}\right)^{1 / n}+(1-\eta) \frac{E^{2}}{\rho_{m}}, T<T_{c B}, \\
(1-\eta) \frac{E^{2}}{\rho_{m}}, T \geq T_{c B} .
\end{array}\right.
\end{aligned}
$$

При этом напряженность электрического поля как функция температуры для заданного значения плотности тока является решением трансцендентного уравнения

$$
\eta J_{c 0}\left(1-\frac{T-T_{0}}{T_{c B}-T_{0}}\right)\left(\frac{E}{E_{c}}\right)^{1 / n}+(1-\eta) \frac{E}{\rho_{m}}-J=0
$$

при $T<T_{c B}$ и описывается очевидным равенством

$$
E=J \rho_{m} /(1-\eta)
$$

при $T \geq T_{c B}$.

Выполним в рамках сформулированных моделей качественный анализ формирования резистивных состояний сверхпроводящего композита. Для этого предположим, что величина удельного электрического сопротивления матрицы постоянна. Введем следующие безразмерные переменные: $i=J /\left(\eta J_{c 0}\right)$ - безразмерный ток, $e=E / E_{c}-$ безразмерная напряженность электрического поля, $\theta=\left(T-T_{0}\right) /\left(T_{c B}-T_{0}\right)-$ безразмерная температура, $g_{\mathrm{CSM}}=G_{\mathrm{CSM}} /\left(\eta J_{c 0} E_{c}\right)-$ безразмерное тепловыделение, вычисленное в рамках $\mathrm{MKC}$, $g=G /\left(\eta J_{c 0} E_{c}\right)$ - безразмерное тепловыделение, вычисленное с учетом ВАХ. Тогда соотношения (1) и (4) преобразуются к виду

$$
g_{\mathrm{CSM}}(\theta)=i^{2} \varepsilon_{1}\left\{\begin{array}{l}
1, \theta>1, \\
(\theta-1+i) / i, \quad 1-i \leq \theta \leq 1, \\
0, \theta<1-i
\end{array}\right.
$$


для модели критического состояния и

$$
g(\theta)=\left\{\begin{array}{l}
(1-\theta) e^{1+1 / n}+e^{2} / \varepsilon_{1}, \quad \theta<1, \\
e^{2} / \varepsilon_{1}, \quad \theta \geq 1
\end{array}\right.
$$

для модели со степенной ВАХ. При этом соответствующие безразмерные значения напряженности электрического поля описываются выражениями

$$
\begin{aligned}
e_{\mathrm{CSM}} & =i \varepsilon_{1}\left\{\begin{array}{l}
1, \theta>1, \\
(\theta-1+i) / i, \quad 1-i \leq \theta \leq 1, \\
0, \theta<1-i,
\end{array}\right. \\
i & =\left\{\begin{array}{l}
(1-\theta) e^{1 / n}+e / \varepsilon_{1}, \quad \theta<1, \\
e / \varepsilon_{1}, \theta \geq 1 .
\end{array}\right.
\end{aligned}
$$

Здесь $\varepsilon_{1}=E_{1} / E_{c}$, где $E_{1}=\eta J_{c 0} \rho_{m} /(1-\eta)$.

$\mathrm{BAX}(9)$ и (10) позволяют найти безразмерное эффективное электрическое сопротивление композита, если воспользоваться равенствами $e_{\mathrm{CSM}}=i \varepsilon_{1} r_{\mathrm{CSM}}, e=i \varepsilon_{1} r_{k}$ для каждой из рассматриваемой модели.

Переход к безразмерным переменным показывает существование характерного безразмерного параметра $\varepsilon_{1}$, значение которого зависит от свойств сверхпроводника и матрицы. Оценим его для высокотемпературного сверхпроводника, покрытого серебром, полагая, что $\rho_{m} \sim 10^{-7} \Omega \mathrm{cm}, J_{c 0} \sim 10^{5} \mathrm{~A} / \mathrm{cm}^{2}, \quad \eta \sim 0.5$, $T_{c B}-T_{0} \sim 20 \mathrm{~K}, E_{c} \sim 10^{-6} \mathrm{~V} / \mathrm{cm}$. Тогда $\varepsilon_{1} \sim 10^{4}$. Он позволяет оценить влияние сопротивления матрицы на процесс деления тока между сверхпроводником и матрицей для композитных проводников с реальной ВАХ. Действительно, согласно (10), при $\varepsilon_{1} \gg 1$ основная часть тока будет течь по сверхпроводнику, если температура композита незначительно отличается от температуры хладагента. В то же время в теории тепловой стабилизации, основанной на МКС, имеет место другой механизм деления тока. Он начинает перетекать в матрицу после того, когда транспортный ток превысит критический ток композита. Поэтому процесс перераспределения тока между сверхпроводником и матрицей не зависит от ее сопротивления, т. е. от величины $\varepsilon_{1}$. Для иллюстрации роли $\varepsilon_{1}$ в формировании токовых состояний технического сверхпроводника на рис. 1 между собой сопоставлены результаты расчета процесса деления тока между сверхпроводником и матрицей, полученные на основании использования обеих моделей при $i=0.8$. Моделирование токовых состояний композита со степенной ВАХ было выполнено, согласно (10), при $n=25$ и различных значениях параметра $\varepsilon_{1}$. В рамках МКС безразмерные токи в сверхпроводнике $i_{s c}$ и матрице $i_{m}$ в диапазоне температур $1-i<\theta<1$ рассчитывались по очевидным зависимостям [1]: $i_{s c}=1-\theta, i_{m}=i-1+\theta$. Рисунок наглядно демонстрирует отличие в процессе деления тока, описываемый сформулированными выше моделями, и его зависимость от $\varepsilon_{1}$.

На рис. 2,3 приведены зависимости эффективного электрического сопротивления сверхпроводящего композита от температуры, рассчитанные согласно (9)

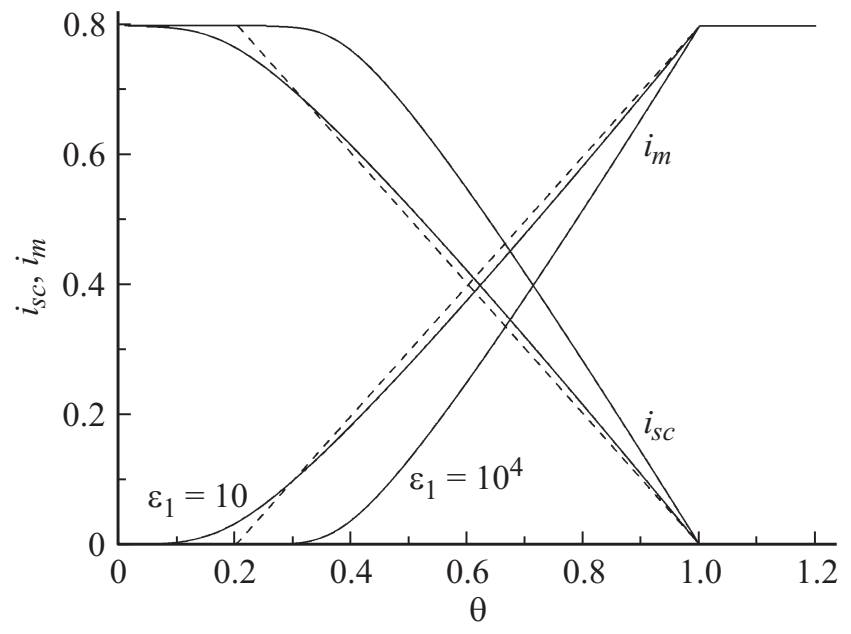

Рис. 1. Влияние параметра $\varepsilon_{1}$ на деление тока между сверхпроводником и матрицей: - - модель со степенной $\mathrm{BAX}$, -- - МКC.

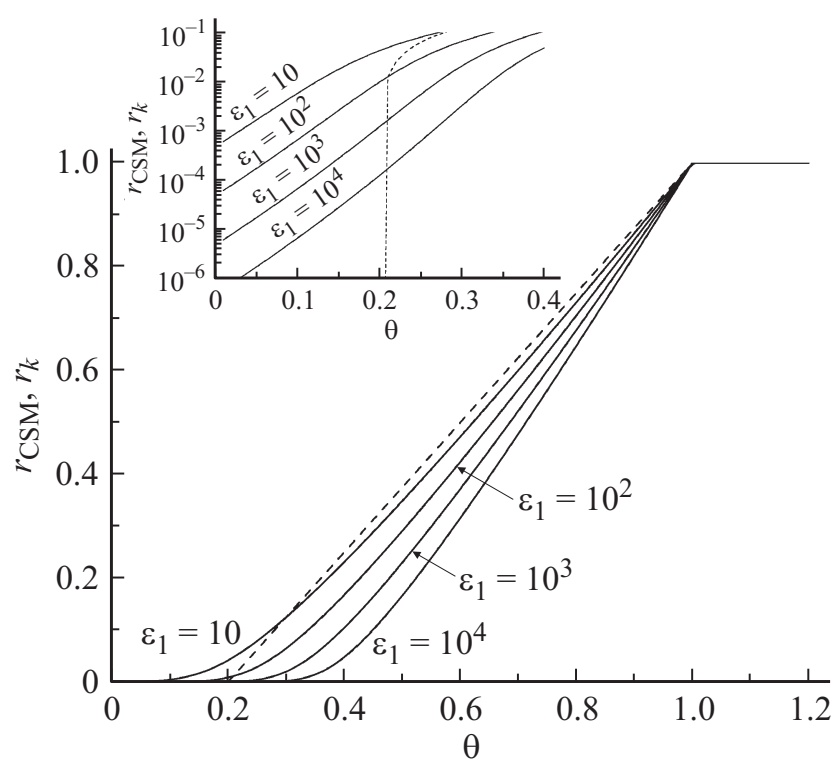

Рис. 2. Температурные зависимости эффективного электрического сопротивления композитного сверхпроводника при $i=0.8$ и различных значениях параметра $\varepsilon_{1}:-$ модель со степенной ВАХ, --- - МКС.

и (10). В рамках приближения (10) вычисления были выполнены при $n=25$ и различных значениях параметра $\varepsilon_{1}$ и транспортного тока. А именно рис. 2 демонстрирует влияние параметра $\varepsilon_{1}$ на значения $r_{\operatorname{CSM}}(\theta)$ и $r_{k}(\theta)$ при токе, меньшем критического. Вставка к рис. 2 показывает соответствующие значения $r_{\mathrm{CSM}}(\theta)$ и $r_{k}(\theta)$ в области малых перегревов, в которой имеется общая точка, где кривые $r_{\operatorname{CSM}}(\theta)$ и $r_{k}(\theta)$ пересекаются. На рис. 3 изображены температурные зависимости эффективного электрического сопротивления композита при токах, превышающих критический ток композита. В рамках МКС весь ток при $i \geq 1$ будет протекать 


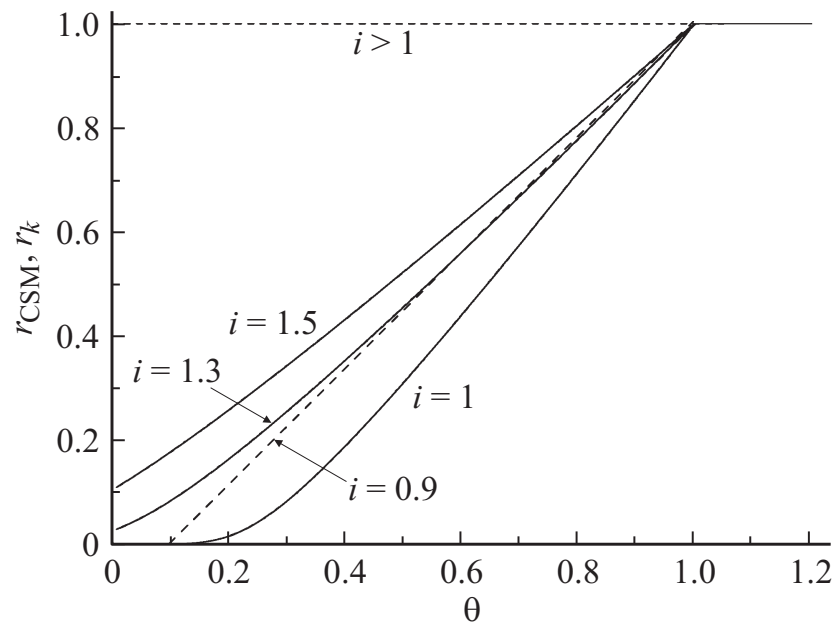

Рис. 3. Температурные зависимости эффективного электрического сопротивления композитного сверхпроводника при $\varepsilon_{1}=10^{4}$ и токах, превышающих критическое значение: - модель со степенной ВАХ, --- - МКС

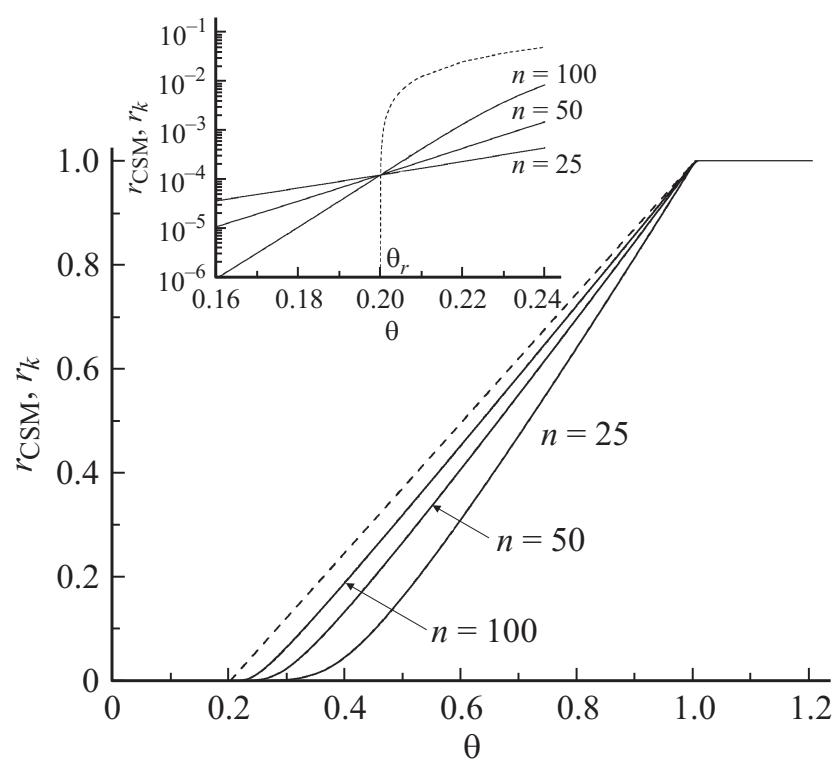

Рис. 4. Влияние параметра нарастания ВАX сверхпроводника на эффективное электрическое сопротивление композита при $i=0.8$ и $\varepsilon_{1}=10^{4}:-$ модель со степенной $\mathrm{BAX},---$ МKC.

только по матрице в силу разрушения сверхпроводящего состояния. Отличия между значениями $r_{\operatorname{CSM}}(\theta)$ и $r_{k}(\theta)$, обусловленные влиянием параметра крипа, представлены на рис. 4. Здесь же на вставке к рисунку эти значения показаны более детально в области перегревов, близких к температуре резистивного перехода. Состояния, следующие из модели со степенной ВАХ, изображены на рис. 2-4 сплошными линиями. Штриховые линии соответствуют состояниям, которые описываются МКС. Представленные результаты позволяют сделать следующие выводы.
В диапазоне температур от 0 до $\theta=1-i$ значения $r_{k}(\theta)$ отличны от нуля, в то время как значения $r_{\operatorname{CSM}}(\theta)$ равны нулю. При этом разница между ними возрастает с увеличением тока при уменьшении $n$ и $\varepsilon_{1}$. Данное отличие обусловлено малым, но конечным значением напряженности электрического поля, возникающим в композитном сверхпроводнике с реальной ВАХ.

При $\theta>1-i$ существует температура $\theta_{r}=1-i+$ $+1 / \varepsilon_{1}$ (в размерном виде $\left.T_{t}=T_{c s}+\left(T_{c B}-T_{0}\right) / \varepsilon_{1}\right)$, при которой значения $r_{\mathrm{CSM}}(\theta)$ и $r_{k}(\theta)$ принимают равные значения. Как следует из (9) и (10), в этом случае $e_{\operatorname{CSM}}\left(\theta_{r}\right)=e\left(\theta_{r}\right)=1$, т. е. напряженность электрического поля равна заданному критическому значению $\left(E=E_{c}\right)$. Следовательно, уже при незначительном перегреве композита относительно температуры резистивного перехода, равном $1 / \varepsilon_{1}$, значения напряженности электрического поля оказываются закритическими даже при докритических значениях введенного в композит тока. При этом в диапазоне температур $\theta_{r}<\theta<1$ значения $r_{\mathrm{CSM}}(\theta)$ всегда выше соответствующих значений $r_{k}(\theta)$, вычисленных для композита со степенной $\mathrm{BAX}$, как это следует из рис. 2,3. Воспользовавшись соотношениями (9) и (10), можно строго доказать, что данная закономерность соблюдается для любого конечного значения $n$. Но при этом будет выполняться предельный переход $e(\theta) \rightarrow e_{\mathrm{CSM}}(\theta)$ при $n \rightarrow \infty$. Поэтому тепловыделения $g_{\mathrm{CSM}}(\theta)$, вычисленные при $\theta_{r}<\theta<1$ в рамках МКС, будут всегда превышать соответствующие тепловыделения $g(\theta)$, реально существующие в сверхпроводящем композите. Из рис. 3 следует, что тепловыделения при закритических токах, вычисленные для композита с непрерывно нарастающей ВАХ, могут быть меньше тепловыделений, вычисленных в рамках МКС при докритическом токе. Поэтому у сверхпроводников с непрерывно нарастающей BAX возможно существование как докритических, так и закритических устойчивых состояний. (Расчеты показывают, что закритические состояния будут заметно расширять диапазон устойчивых токовых режимов при интенсивном охлаждении композитов. Однако их обсуждение выходит за рамки настоящей работы, и они будут изложены отдельно.) Наличие устойчивых докритических и закритических режимов приводит к понятию о предельном токе разрушения сверхпроводимости. Его значение будет являться следствием нарушения теплового баланса между количеством теплоты, выделяемым в композите, и количеством теплоты, отводимым в хладагент [9]. Эти выводы не могут быть сформулированы в рамках МКС.

И, наконец, в области высоких перегревов композита $\left(\theta>1, T>T_{c B}\right)$ значения сопротивлений, рассчитанные как по (9), так и по (10), не отличаются друг от друга в силу принятых выше допушений. В этом случае джоулевы потери, вычисленные в рамках обеих моделей, будут определяться тепловыделениями только в матрице.

Оценим интенсивность нарастания с температурой эффективного электрического сопротивления композита со степенной BAX в диапазоне температур $0<\theta<1$. 
Для начала рассмотрим начальный участок зависимости $r_{k}(\theta)(\theta \ll 1)$. При $\varepsilon_{1} \gg 1$ ток в композите практически равен току в сверхпроводнике и поэтому, согласно (10), получим

$$
i \varepsilon_{1} r_{k}(\theta) \cong\left(\frac{i}{1-\theta}\right)^{n}
$$

Логарифмируя данное равенство, найдем

$$
\ln i \varepsilon_{1} r_{k}(\theta)=n[\ln i-\ln (1-\theta)] .
$$

Раскладывая $\ln (1-\theta)$ в степенной ряд, перепишем последнее равенство в виде

$$
\ln i \varepsilon_{1} r_{k}(\theta)=n\left[\ln i+\theta+\theta^{2} / 2+\ldots\right] .
$$

Тогда

$$
r_{k}(\theta)=\frac{i^{n-1}}{\varepsilon_{1}} \exp \left[n\left(\theta+\theta^{2} / 2+\ldots\right)\right] .
$$

Следовательно, нарастание с температурой начального участка зависимости $r_{k}(\theta)$ носит экспоненциальный характер. Причем скорость нарастания $d r_{k} / d \theta$ тем больше, чем выше $n$. Данные выводы подтверждают вставки к рис. $2,4$.

Для оценки $r_{k}(\theta)$ вблизи критической температуры композита введем новую функцию $u=1-r_{k}$, которая, согласно (12), приведет к уравнению

$$
1=\frac{1-\theta}{i}\left[(1-u) i \varepsilon_{1}\right]^{1 / n}+1-u .
$$

Раскладывая в степенной ряд сомножитель $(1-u)^{1 / n}$, получим

$$
1=\frac{1-\theta}{i}\left(i \varepsilon_{1}\right)^{1 / n}\left(1-\frac{u}{n}-\frac{n-1}{2 n^{2}} u^{2}+\ldots\right)+1-u .
$$

Тогда, ограничиваясь линейным по температуре приближением, найдем

$$
r_{k}(\theta)=1-\frac{1}{i}\left(i \varepsilon_{1}\right)^{1 / n}+\frac{\theta}{i}\left(i \varepsilon_{1}\right)^{1 / n},
$$

т.е. вблизи критической температуры сверхпроводника температурное нарастание эффективного электрического сопротивления композита со степенной BAX происходит практически линейно. Как следует из рис. 2-4, данная закономерность будет соблюдаться тем лучше, чем выше $i$ и $n$. Отметим, что она имеет место в силу сделанных выше упрощающих допущений и прежде всего в случаях, когда критическая плотность тока сверхпроводника является линейной функцией температуры.

Переход от экспоненциального роста с температурой эффективного электрического сопротивления композита с реальной ВАХ к линейному наглядно демонстрирует стабилизирующую роль матрицы в его тепловой стабилизации. Очевидно, правомерен более общий вывод: чем ближе при повышении температуры эффективное сопротивление композита с реальной ВАХ к линейной зависимости, тем больше ток в матрице. Модель критического состояния не позволяет учесть отмеченные особенности нарастания с температурой эффективного электрического сопротивления композита, поскольку в еe рамках зависимость джоулева тепловыделения от температуры (1) линейна в соответствии с уменьшением критического тока композита.

В основе обсужденных выше закономерностей лежат особенности изменения дифференциального сопротивления сверхпроводника в зависимости от характера нарастания с температурой его ВАХ как при докритических значениях напряженности электрических полей $(e<1)$, так и при их закритических значениях $(e>1)$. Действительно, согласно рис. 4, даже при очень высоком, но конечном значении $n$, например $n=100$, в температурных зависимостях эффективного значения сопротивления композита со степенной BAX также имеют место отмеченные особенности его нарастания. Отличие между расчетными значениями по обеим моделям увеличивается с уменьшением параметра $n$. В результате модель со степенной ВАХ будет приводить к более заниженным значениям тепловыделений в диапазоне температур $\theta_{r}<\theta<1$. Важно подчеркнуть, что данные закономерности будут иметь место так же и в токонесущих элементах на основе низкотемпературных сверхпроводников, для которых $n>50$. Поэтому теоретический анализ условий тепловой стабильности сверхпроводников, выполненный в рамках МКС, будет неизбежно приводить к заниженным значениям критических энергий внешних тепловых возмущений и к завышенным оценкам скорости необратимого распространения тепловой неустойчивости при ее появлении в реальных токонесущих элементах. В то же время, поскольку в области температур, превышающих критическую температуру сверхпроводника, отличия между рассматриваемыми моделями отсутствуют, то они будут приводить к одинаковым оценкам условий пережога технических сверхпроводников.

Рис. 5 демонстрирует особенности деления тока между сверхпроводником и матрицей при докритическом и закритических токах при $n=25$ и $\varepsilon_{1}=10^{4}$. Здесь штриховая прямая показывает безразмерную температурную зависимость критического тока композита $i_{c}$, а сплошные и штрихпунктирные кривые - токи в сверхпроводнике $\left(i_{s c}\right)$ и матрице $\left(i_{m}\right)$ соответственно. Видно, что при докритическом токовом режиме (кривая 1) интенсивное деление тока в композите с реальной ВАХ не наблюдается, даже когда его температура превышает температуру резистивного перехода $(\theta=0.2)$. Более того, ток в сверхпроводящей части композита остается выше критического тока композитного сверхпроводника и после значительного превышения температуры резистивного перехода. При закритических режимах ток в сверхпроводнике также всегда больше критического тока композита даже при повышении его температуры вплоть до критической температуры сверхпроводника. Следовательно, температура резистивного перехода $T_{c s}$, 


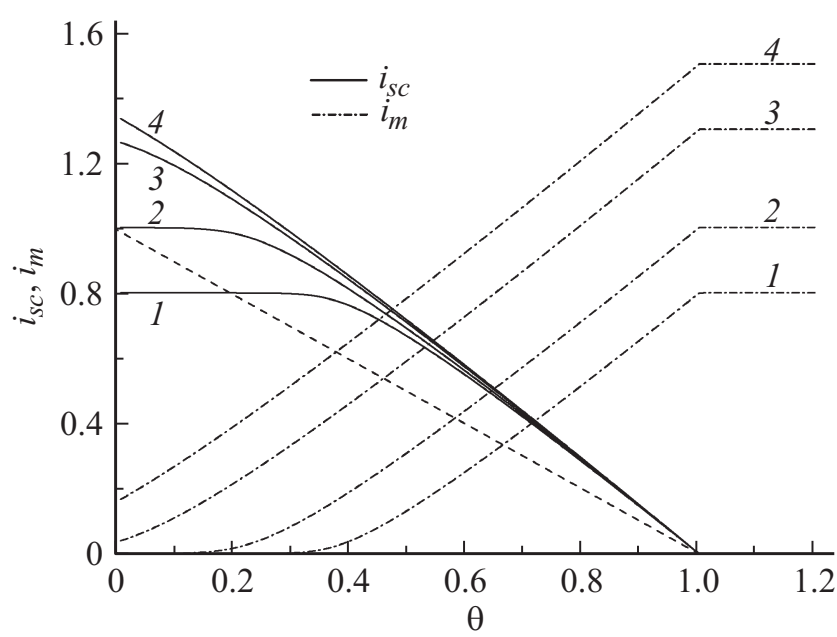

Pис. 5. Особенности деления тока между сверхпроводником и матрицей при различных значениях транспортного тока $i$ : $1-0.8,2-1,3-1.3,4-1.5 ;-,-\cdot-\cdot-$ - модели со степенной ВАХ, -- - - критический ток сверхпроводника.

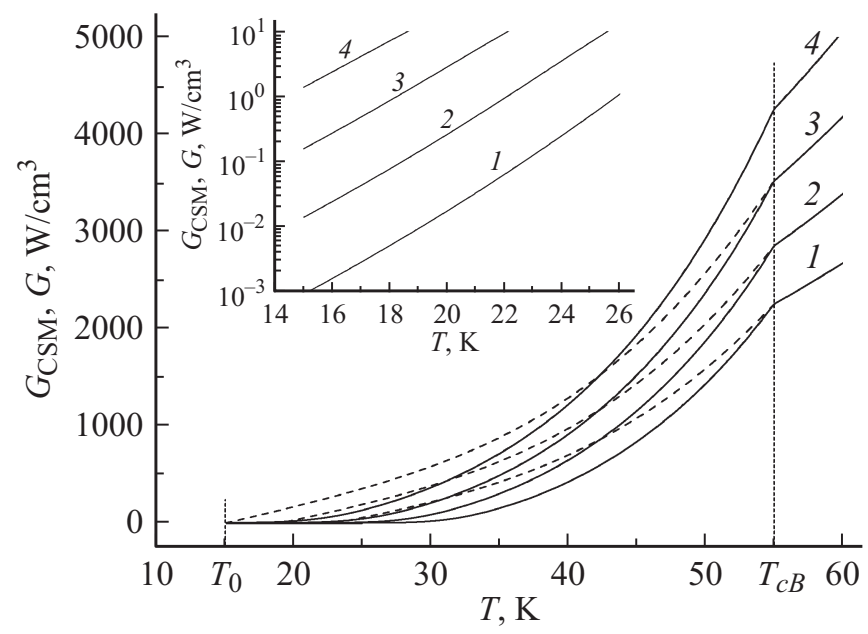

Рис. 6. Температурные зависимости джоулева тепловыделения при докритических и закритических токах при $T_{0}=15 \mathrm{~K}, I$ : $1-160,2-180,3-199,4-220$ A.

после которой, согласно МКС, начинается процесс деления тока, для композитных сверхпроводников с реальной BAX не имеет физического смысла.

Обсужденные выше характерные закономерности формирования резистивных состояний композитных сверхпроводников, сформулированные в рамках безразмерных моделей (9) и (10), соблюдаются и при анализе джоулевых потерь энергии в реальных сверхпроводящих композитах, электрическое сопротивление матрицы которых зависит от температуры. На рис. 6,7 представлены результаты численного моделирования тепловыделений в сверхпроводящей ленте на основе YВСО с двумя стабилизирующими покрытиями из серебра и меди, описанной в [10]. Соответственно ее геометрические параметры (ширина $b$, толщина сверх- проводника $a_{s}$, толщина серебра $a_{A g}$ и толщина меди $\left.a_{C u}\right)$ принимались равными $b=0.2 \mathrm{~cm}, a_{s}=10^{-4} \mathrm{~cm}$, $a_{A g}=17 \cdot 10^{-4} \mathrm{~cm}, a_{C u}=17 \cdot 10^{-4} \mathrm{~cm}$, плотность критического тока сверхпроводника была описана линейной зависимостью (2) с критическими параметрами $I_{c 0}=200 \mathrm{~A}$ и $T_{c B}=55 \mathrm{~K}$ во внешнем магнитном поле $B=10 \mathrm{~T}$ и при температуре охладителя $T_{0}=15 \mathrm{~K}$. Параметры ВАХ задавались равными $E_{c}=10^{-6} \mathrm{~V} / \mathrm{cm}$, $n=22$. Для расчета удельного электрического сопротивления серебра и меди использовались результаты $[11-13]$, принимая $\rho_{A g}(273 \mathrm{~K})=1.48 \cdot 10^{-6} \Omega \cdot \mathrm{cm}$ для серебра и $\rho_{C u}(273 \mathrm{~K})=1.55 \cdot 10^{-6} \Omega \cdot \mathrm{cm}$ для меди при $R R R=\rho(273 \mathrm{~K}) / \rho(4.2 \mathrm{~K})=100$ как для серебра, так и для меди. Тепловыделение в ленте в рамках МКС рассчитывалось по формуле

$$
\begin{aligned}
& G_{\mathrm{CSM}}(T)=\frac{I^{2}}{S^{2}} \rho_{k}(T, B) \\
& \times\left\{\begin{array}{l}
1, T>T_{c B}, \\
\left(T-T_{c s}\right) /\left(T_{c B}-T_{c s}\right), T_{c s} \leq T \leq T_{c B}, \\
0, T<T_{c s}=T_{c B}-\left(T_{c B}-T_{0}\right) I / I_{c 0},
\end{array}\right.
\end{aligned}
$$

где

$$
\rho_{k}(T, B)=\frac{\left(a_{s}+a_{A g}+a_{C u}\right) \rho_{A g}(T, B) \rho_{C u}(T, B)}{a_{C u} \rho_{A g}(T, B)+a_{A g} \rho_{C u}(T, B)} .
$$

Формула (4) для данной ленты приводится к виду

$$
G(T)=\left\{\begin{array}{l}
\frac{I_{c 0} E}{S} \frac{T_{c B}-T}{T_{c B}-T_{0}}\left(\frac{E}{E_{c}}\right)^{1 / n}+\frac{E^{2}}{\rho_{k}}, \quad T<T_{c B}, \\
\frac{E^{2}}{\rho_{k}}, \quad T \geq T_{c B} .
\end{array}\right.
$$

Состояния, следующие из МКС, на рис. 6, 7 изображены штрихом. Сплошные линии описывают тепловыделения, рассчитанные для ленты со степенной ВАХ. Coответствующие температурные зависимости $G_{\mathrm{CSM}}$ и $G$

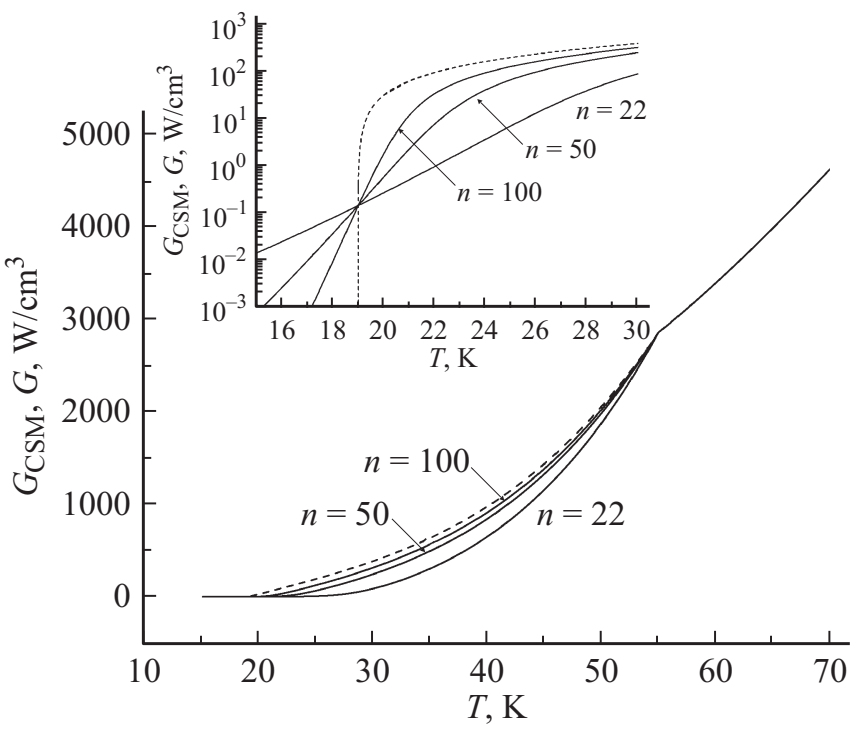

Рис. 7. Влияние параметра нарастания ВАХ на джоулево тепловыделение в сверхпроводящей ленте при $I=180$ А. 


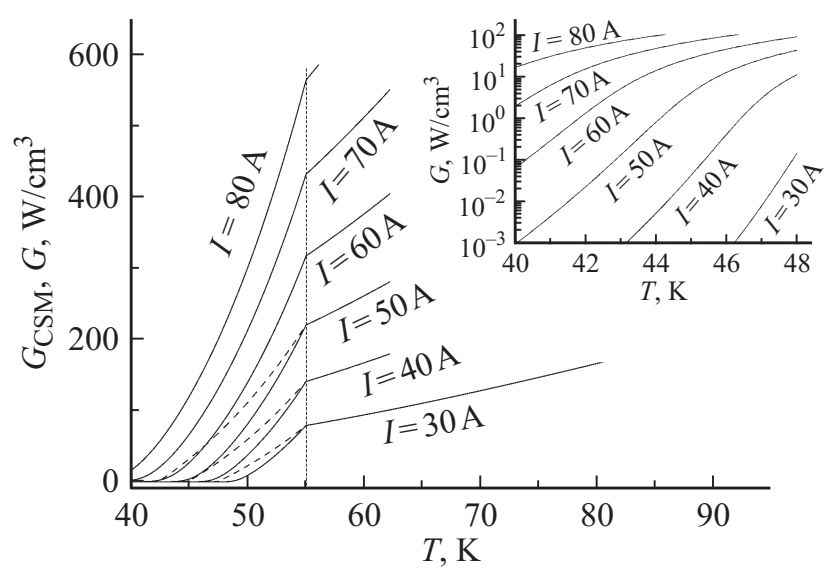

Рис. 8. Температурные зависимости джоулева тепловыделения при докритических и закритических токах при $T_{0}=40 \mathrm{~K}$.

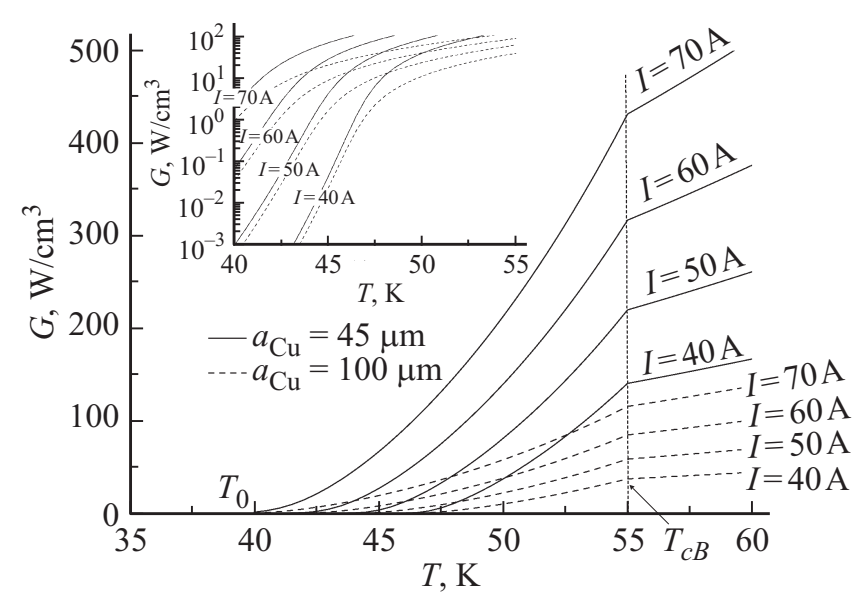

Рис. 9. Влияние толщины медного покрытия на джоулево тепловыделение в сверхпроводящей ленте.

при малых перегревах более детально изображены на вставках к рис. 6,7 .

На рис. 8,9 и на вставках к ним показаны тепловыделения в той же ленте как функции температуры, вычисленные при $T_{0}=40 \mathrm{~K}$. В этом случае критический ток ленты равен $59 \mathrm{~A}$.

Рис. 6-9 наглядно демонстрируют, что МКС не позволяет корректно описать диссипативные явления в реальных сверхпроводящих композитах в силу заложенного в нее механизма скачкообразного перехода из сверхпроводящего состояния в нормальное. А именно МКС приводит не только к завышенным тепловыделениям в диапазоне температур $T_{r}<T<T_{c B}$, но и не описывает при $T_{r}<T<T_{C B}$ их реальное нарастание с температурой, когда экспоненциальный рост $G(T)$ по мере увеличения температуры хладагента или толщины стабилизирующего покрытия перестает быть столь интенсивным, приближаясь практически к линейной зависимости.

Таким образом, крип магнитного потока оказывает существенное влияние на происходящие в композитных сверхпроводниках диссипативные явления. В результате его учет в теории тепловой стабилизации сверхпроводящих композитов неизбежно приведет не только к количественным отличиям от результатов, которые следуют из существующей теории тепловой стабилизации, но и к качественно новым выводам. Во-первых, у композитных сверхпроводников допустимый диапазон токов не будет ограничиваться величиной критического тока сверхпроводника, поскольку возможно существование устойчивых закритических режимов $(i>1)$. Более того, понятие температуры деления тока так же не имеет физического смысла. Причем в композите с реальной $\mathrm{BAX}$ деление тока происходит таким образом, что ток в сверхпроводнике всегда больше критического тока даже при закритических режимах. Во-вторых, анализ условий стабильности сверхпроводящего состояния по отношению к внешним тепловым возмущениям будет приводить не только к более оптимистическим оценкам допустимых значений энергий возмущений, инициирующих тепловую неустойчивость, но и к их конечным значениям в области закритических токов. Последние в рамках МКС равны нулю для всех $i \geq 1$. В-третьих, скорости необратимого распространения тепловых неустойчивостей вдоль композитного сверхпроводника будут тем меньше, чем меньше параметр крипа в силу соответствующего уменьшения джоулева тепловыделения.

Обсужденные в настоящей работе особенности формирования резистивных состояний сверхпроводящих композитов необходимо учитывать при анализе условий тепловой стабильности токонесущих элементов как на основе низкотемпературных, так и высокотемпературных сверхпроводников.

\section{Список литературы}

[1] Альтов В.А., Зенкевич В.Б., Кремлев М.Г., Сычев В.В. Стабилизация сверхпроводящих магнитных систем. М.: Энергоатомиздат, 1984. 312 с.

[2] Уилсон М. Сверхпроводящие магниты. М.: Мир, 1985. $407 \mathrm{c}$.

[3] Гуревич А.Вл., Мини Р.Г., Рахманов А.Л. Физика композитных сверхпроводников. М.: Наука, 1987. 240 с.

[4] Dresner L. // Cryogenics. 1993. Vol. 33. N 9. P. 900-909.

[5] Lim H., Iwasa Y. // Cryogenics. 1997. Vol. 37. N 12. P. 789799.

[6] Tsukamoto O., Fujimoto Y., Takao T. // Cryogenics. 2014. Vol. 63. P. 148-154.

[7] Zeldov E., Amer N.M., Koren G. et al. // Appl. Phys. Lett. 1990. Vol. 56. N 7. P. 680-682.

[8] Edelman H.S., Larbalestier D.C. // J. Appl. Phys. 1993. Vol. 74. N 5. P. 3312-3315.

[9] Polak M., Hlasnik I., Krempasky L. // Cryogenics. 1973. Vol. 13. N 12. P. 702-711.

[10] Awaji S., Hou Y., Oguro H., Watanabe K. et al. // IEEE Trans. Appl. Supercon. 2012. Vol. 22. N 3. P. 6601004.

[11] Seeber B. Handbook of Applied Superconductivity / Ed. by B. Seeber. Bristol: IOP Publishing, 1998. Vol. 1. P. 1067-1082. 